\title{
Quantum information processing and composite quantum fields
}

\author{
Sanjaye Ramgoolam ${ }^{a, b}$ and Michal Sedlák ${ }^{c, d}$ \\ ${ }^{a}$ Centre for Research in String Theory, \\ Department of Physics, Queen Mary University of London, \\ Mile End Road, London E1 4NS, U.K. \\ ${ }^{b}$ National Institute for Theoretical Physics, \\ School of Physics and Mandelstam Institute for Theoretical Physics, \\ University of the Witwatersrand, Wits, 2050, South Africa \\ ${ }^{c} R C Q I$, Institute of Physics, Slovak Academy of Sciences, \\ Dubravska cesta 9, 84511 Bratislava, Slovakia \\ ${ }^{d}$ Faculty of Informatics, Masaryk University, \\ Botanická 68a, 60200 Brno, Czech Republic \\ E-mail: s.ramgoolam@qmul.ac.uk, fyzimsed@savba.sk
}

ABSTRACT: Some beautiful identities involving hook contents of Young diagrams have been found in the field of quantum information processing, along with a combinatorial proof. We here give a representation theoretic proof of these identities and a number of generalizations. Our proof is based on trace identities for elements belonging to a class of permutation centralizer algebras. These algebras have been found to underlie the combinatorics of composite gauge invariant operators in quantum field theory, with applications in the AdS/CFT correspondence. Based on these algebras, we discuss some analogies between quantum information processing tasks and the combinatorics of composite quantum fields and argue that this can be fruitful interface between quantum information and quantum field theory, with implications for AdS/CFT.

KeYwords: 1/N Expansion, AdS-CFT Correspondence

ArXiv EPRINT: 1809.05156 


\section{Contents}

1 Identities from quantum information theory 1

2 Representation theoretic proof $\quad 1$

2.1 Trace of a sum of permutations in $V_{N}^{\otimes(n+1)} 3$

3 A generalization with $S_{m} \times S_{n} \quad 4$

4 Multi-partite generalization $\quad 7$

5 Permutation centralizer algebras, composite gauge invariant operators and AdS/CFT $\quad 9$

A some facts about $\mathrm{U}(N), S_{n}$ and the tensor product $V_{N}^{\otimes n}$

\section{Identities from quantum information theory}

Some beautiful symmetric group identities have been found in the subject of quantum information processing [1]. A combinatoric proof has been given [2].

The key identity is

$$
d_{r} n(n+1)=\sum_{R \vdash(n+1)} d_{R} g(r, \square, R)\left(c_{\square}(R, r)\right)^{2},
$$

where $r$ is a Young diagram with $n$ boxes. $R$ is a Young diagram with $n+1$ boxes, $d_{r}$ is the dimension of the irreducible representation (irrep) of the symmetric group $S_{n}$ associated with the Young diagram $r, d_{R}$ is the dimension of the $S_{n+1}$ irrep associated with $R$. Finally, $g(r, \square, R)$ is the Littlewood-Richardson coefficient coupling the $V_{r}^{U} \otimes V_{\square}^{U}$ with $V_{R}^{U}$, where $V_{r}^{U}, V_{\square}^{U}, V_{R}^{U}$ are the $\mathrm{U}(N)$ (or GL(N)) irreps associated with the respective Young diagrams and $c_{\square}(R, r)$ is the content (difference of the column and row number) of the additional box in Young diagram $R$ which is not contained in $r$.

\section{Representation theoretic proof}

Consider the tensor product of $\mathrm{U}(N)$ irreps.

$$
V_{r}^{U} \otimes V_{\square}^{U}
$$

It is an irredicible representation of $\mathrm{U}(N) \times \mathrm{U}(N)$. Under the diagonal subgroup, it is reducible. The decomposition is given by

$$
V_{r}^{U} \otimes V_{\square}^{U}=\bigoplus_{R \vdash n+1} g(r, \square, R) V_{R}^{U}
$$


It follows that

$$
N \operatorname{Dim}_{N}(r)=\sum_{R \vdash n+1} g(r, \square, R) \operatorname{Dim}_{N} R
$$

We also have a representation of $S_{n} \times S_{1}$ associated with $(r, \square) . S_{n} \times S_{1}$ is a subgroup of $S_{n+1}$. We can induce a representation of $S_{n+1}$ from this representation of $S_{n} \times S_{1}$. This is a reducible representation of $S_{n+1}$. The multiplicity of an irrep $R$ in this reducible rep is $g(r, \square, R)$ (see for example [3]).

This means that

$$
(n+1) d_{r}=\sum_{R \vdash(n+1)} g(r, \square, R) d_{R}
$$

On the l.h.s. we used the fact that the dimension of the rep of $S_{n+1}$ induced from the irrep $V_{r}^{S_{n}} \otimes V_{\square}^{S_{1}}$ of $S_{n} \times S_{1}$, is

$$
\frac{\left|S_{n+1}\right|}{\left|S_{n} \times S_{1}\right|} \operatorname{Dim}\left(V_{r}^{S_{n}} \otimes V_{\square}^{S_{1}}\right)=\frac{(n+1) !}{n !} d_{r}=(n+1) d_{r}
$$

On the r.h.s. we use the decomposition in terms of irreps of $S_{n+1}$.

Useful relation between $\operatorname{Dim}_{N} r$ and $d_{r}$ is

$$
\operatorname{Dim}_{N} r=\frac{d_{r} f_{r}}{n !},
$$

where $f_{r}$ is the product over the boxes of the Young diagram of $\left(N-c_{\square}\right)$ and $c_{\square}=(i-j)$ for a box at row $i$ and column $j$.

$$
f_{r}=\prod_{\square \in r}\left(N-c_{\square}\right)=\prod_{i, j}(N+j-i)
$$

Similarly,

$$
\operatorname{Dim}_{N} R=\frac{d_{R} f_{R}}{(n+1) !}
$$

Thus the ratio reads

$$
\frac{\operatorname{Dim}_{N} R}{\operatorname{Dim}_{N} r}=\frac{d_{R}}{d_{r}(n+1)}\left(N-c_{\square}(R, r)\right),
$$

where $c_{\square}(R, r)$ is the content of the box by which $R$ and $r$ differ. We can also write $c_{\square}(R, r)=\sum_{\square \in R} c_{\square}(R)-\sum_{\square \in r} c_{\square}(r)$. Using the above equation in (2.3) we have

$$
N=\sum_{R \vdash(n+1)} g(r, \square, R) \frac{d_{R}}{d_{r}(n+1)}\left(N-c_{\square}(R, r)\right)
$$

Comparing this with the induction equation (2.4), we find

$$
\sum_{R \vdash(n+1)} g(r, \square, R) c_{\square}(R, r) d_{R}=0 .
$$




\subsection{Trace of a sum of permutations in $V_{N}^{\otimes(n+1)}$}

In the group algebra $\mathbb{C}\left(S_{n+m}\right)$, an interesting sub-algebra is formed by the subspace which is invariant under conjugation by elements of $S_{n} \times S_{m}$. This is an example of what was called a permutation centralizer algebra (PCA) in $[4,5]$, and which has many applications in the study of gauge invariant operators with relevance to AdS/CFT. It is denoted $\mathcal{A}(n, m)$. For $m=1$ we have $\mathcal{A}(n, 1)$. The element $\sum_{a=1}^{n}(a, n+1)$, which we will use here, is an element of $\mathcal{A}(n, 1)$. It is very interesting that PCAs are also finding a use in quantum information processing (QIP). We discuss this further in section 5.

The tensor product $V_{r} \otimes V_{\square}$ is a subspace of $V_{N}^{\otimes(n+1)}$, where $V_{N}$ is the fundamental of $\mathrm{U}(N)$.

$$
\begin{aligned}
W=V_{N}^{\otimes n+1} & =V_{N}^{\otimes n} \otimes V_{N} \\
& =\bigoplus_{r \vdash n}\left(V_{r}^{U} \otimes V_{r}^{S_{n}}\right) \otimes\left(V_{\square}^{U} \otimes V_{\square}^{S_{1}}\right)
\end{aligned}
$$

Consider the projector $P_{r}$ in the group algebra of $S_{n}$, denoted $\mathbb{C}\left(S_{n}\right)$

$$
P_{r}=\frac{d_{r}}{n !} \sum_{\sigma \in S_{n}} \chi_{r}(\sigma) \sigma
$$

We choose an embedding $\mathbb{C}\left(S_{n}\right) \rightarrow \mathbb{C}\left(S_{n+1}\right)$, where $S_{n}$ acts on $\{1,2, \cdots, n\}$ and $S_{n+1}$ acts on $\{1, \cdots, n, n+1\}$, and construct an element in $\mathbb{C}\left(S_{n+1}\right)$

$$
\left(\frac{P_{r}}{d_{r}} \otimes 1\right) \sum_{a=1}^{n}(a, n+1)
$$

We then consider the trace in $W$

$$
\operatorname{tr}_{W}\left(\frac{P_{r}}{d_{r}} \otimes 1\right) \sum_{a=1}^{n}(a, n+1)
$$

This is a sum of permutations in $S_{n} \subset S_{n+1}$. Doing the multiplication of $P_{r}$ with $\sum_{a=1}^{n}(a, n+1)$ and taking the trace, we get

$$
n \chi_{r}\left(\frac{1}{n !} \sum_{\sigma} N^{C_{\sigma}} \sigma\right)=n \operatorname{Dim}_{N} r
$$

See appendix eq. (A.10) for the derivation.

Let us consider another way to compute the same trace. We observe that

$$
\sum_{a=1}^{n}(a, n+1)=T_{2}^{\left(S_{n+1}\right)}-T_{2}^{\left(S_{n}\right)},
$$


where $T_{2}^{\left(S_{n}\right)}$ is the sum of all permutations in $S_{n}$ which consist of a single swop. Using eqs. (A.11) and (A.16) we get

$$
\begin{aligned}
\operatorname{tr}_{W}\left(\frac{P_{r}}{d_{r}} \otimes 1\right)\left(T_{2}^{\left(S_{n+1}\right)}-T_{2}^{\left(S_{n}\right)}\right) & =\sum_{R \vdash(n+1)} \operatorname{tr}_{W} P_{R}\left(P_{r} \otimes 1\right)\left(T_{2}^{\left(S_{n+1}\right)}-T_{2}^{\left(S_{n}\right)}\right) \\
& =\sum_{R \vdash(n+1)}\left(\frac{\chi_{R}\left(T_{2}^{\left(S_{n+1}\right)}\right)}{d_{R}}-\frac{\chi_{r}\left(T_{2}^{\left(S_{n}\right)}\right)}{d_{r}}\right) \operatorname{tr}_{W} P_{R}\left(\frac{P_{r}}{d_{r}} \otimes 1\right) \\
& =\sum_{R \vdash(n+1)}\left(-c_{\square}(R, r)\right) g(r, \square, R) \operatorname{Dim}_{N} R
\end{aligned}
$$

We made use of the fact

$$
\operatorname{tr}_{W} P_{R}\left(\frac{P_{r}}{d_{r}} \otimes 1\right)=g(r, \square, R) \operatorname{Dim}_{N} R
$$

This is a special case $k=1, n_{1}=n, n_{2}=1$ of an identity (A.27) derived in appendix A.

Now we have

$$
n \operatorname{Dim}_{N} r=\sum_{R \vdash(n+1)}\left(-c_{\square}(r, R)\right) g(r, \square, R) \operatorname{Dim}_{N} R .
$$

Dividing by $\operatorname{Dim}_{N} r$ on both sides we obtain

$$
\begin{aligned}
n & =\sum_{R \vdash(n+1)}\left(-c_{\square}(r, R)\right) g(r, \square, R) \frac{d_{R}\left(N-c_{\square}(r, R)\right)}{d_{r}(n+1)} \\
& =\sum_{R \vdash(n+1)}-N c_{\square}(r, R) g(r, \square, R) d_{R}+\sum_{R \vdash(n+1)}\left(c_{\square}(r, R)\right)^{2} g(r, \square, R) \frac{d_{R}}{(n+1) d_{r}} .
\end{aligned}
$$

The first term in the above equation is zero due to eq. (2.11). Thus, we get

$$
d_{r} n(n+1)=\sum_{R \vdash(n+1)}\left(c_{\square}(r, R)\right)^{2} g(r, \square, R) d_{R},
$$

which is the desired identity.

\section{A generalization with $S_{m} \times S_{n}$}

Consider $S_{m} \times S_{n} \rightarrow S_{m+n}$ and the vector space $W=V_{N}^{\otimes m+n}$.

For Young diagrams $r, s$ with $m, n$ boxes respectively, consider

$$
V_{r}^{U} \otimes V_{s}^{U}=\bigoplus_{R \vdash m+n} g(r, s, R) V_{R}^{U}
$$

which gives the decomposition into irreducible representations of the $\mathrm{U}(N)$ which acts diagonally on $V_{r}^{U} \otimes V_{s}^{U}$. First consider the dimension on both sides of the equation

$$
\operatorname{Dim}_{N} r \operatorname{Dim}_{N} s=\sum_{R \vdash(m+n)} g(r, s, R) \operatorname{Dim}_{N} R,
$$


which is using eq. (2.6) rewritten as

$$
\frac{(m+n) !}{m ! n !} d_{r} d_{s}=\sum_{R \vdash(m+n)} g(r, s, R) d_{R} \frac{f_{R}}{f_{r} f_{s}}
$$

On the other hand, using induction of $V_{r}^{S_{m}} \otimes V_{s}^{S_{n}}$ from $S_{m} \times S_{n}$ to $S_{m+n}$ we obtain

$$
\frac{(m+n) !}{m ! n !} d_{r} d_{s}=\sum_{R} g(r, s, R) d_{R}
$$

The expression $\frac{f_{R}}{f_{r} f_{s}}$ has a large $N$ expansion $1+\mathcal{O}(1 / N)$. Comparing the two equations, we conclude that all the $1 / N$ corrections in

$$
\frac{f_{R}}{f_{r} f_{s}}=\frac{\prod_{\square \in R}\left(1-\frac{c_{\square}(R)}{N}\right)}{\prod_{\square \in r}\left(1-\frac{c_{\square}(r)}{N}\right) \prod_{\square \in s}\left(1-\frac{c_{\square}(s)}{N}\right)}
$$

lead to identities. For example,

$$
\begin{aligned}
0= & \sum_{R \vdash(m+n)} g(r, s, R) d_{R}\left(-\sum_{\square \in R} c_{\square}(R)+\sum_{\square \in r} c_{\square}(r)+\sum_{\square \in s} c_{\square}(s)\right) \\
0= & \sum_{R \vdash(m+n)} g(r, s, R) d_{R}\left(\sum_{i_{1}<i_{2} \in R} c_{i_{1}}(R) c_{i_{2}}(R)-\sum_{i \in R} \sum_{k \in r} c_{i}(R) c_{k}(r)-\sum_{i \in R} \sum_{l \in s} c_{i}(R) c_{l}(s)\right. \\
& \left.+\sum_{k \in r} \sum_{l \in s} c_{k}(r) c_{l}(s)+\sum_{k \in r}\left(c_{k}(r)\right)^{2}+\sum_{l \in s}\left(c_{l}(s)\right)^{2}+\sum_{k_{1}<k_{2} \in r} c_{k_{1}}(r) c_{k_{2}}(r)+\sum_{l_{1}<l_{2} \in s} c_{l_{1}}(s) c_{l_{2}}(s)\right)
\end{aligned}
$$

There will also be higher order equations: at each order in $1 / N$ the equation involves Littlewood-Richardson coefficients and Young diagram contents, hence just data pertaining to the symmetric groups. All the equations arise from the large $N$ expansion of

$$
0=\sum_{R \vdash(m+n)} g(r, s, R) d_{R}\left(\frac{f_{R}}{f_{r} f_{s}}-1\right) .
$$

Now consider the trace

$$
\operatorname{tr}_{W}\left(\left(\frac{P_{r}}{d_{r}} \otimes \frac{P_{s}}{d_{s}}\right)\left(T_{2}^{\left(S_{m+n}\right)}-T_{2}^{\left(S_{m}\right)}-T_{2}^{\left(S_{n}\right)}\right)\right) .
$$

We observe that

$$
\left(T_{2}^{\left(S_{m+n}\right)}-T_{2}^{\left(S_{m}\right)}-T_{2}^{\left(S_{n}\right)}\right)=\sum_{a=1}^{m} \sum_{b=m+1}^{m+n}(a, b)
$$


It follows that

$$
\begin{aligned}
& \operatorname{tr}_{W}\left(\left(\frac{P_{r}}{d_{r}} \otimes \frac{P_{s}}{d_{s}}\right)\left(T_{2}^{\left(S_{m+n}\right)}-T_{2}^{\left(S_{m}\right)}-T_{2}^{\left(S_{n}\right)}\right)\right) \\
& =\frac{1}{m ! n !} \sum_{\sigma_{1} \in S_{m}} \sum_{\sigma_{2} \in S_{n}} \chi_{r}\left(\sigma_{1}\right) \chi_{s}\left(\sigma_{2}\right) \operatorname{tr}\left(\left(\sigma_{1} \otimes \sigma_{2}\right) \sum_{a=1}^{m} \sum_{b=m+1}^{m+n}(a, b)\right) \\
& =\frac{m n}{m ! n !} \sum_{\sigma_{1} \in S_{m}} \sum_{\sigma_{2} \in S_{n}} \chi_{r}\left(\sigma_{1}\right) \chi_{s}\left(\sigma_{2}\right) N^{C_{\sigma_{1}}+C_{\sigma_{2}}-1} \\
& =N^{-1} m n \operatorname{Dim}_{N} r \operatorname{Dim}_{N} s
\end{aligned}
$$

On the other hand, using (A.11), (A.12) and (A.27), we derive

$$
\begin{array}{r}
\operatorname{tr}_{W}\left(\left(\frac{P_{r}}{d_{r}} \otimes \frac{P_{s}}{d_{s}}\right)\left(T_{2}^{\left(S_{m+n}\right)}-T_{2}^{\left(S_{m}\right)}-T_{2}^{\left(S_{n}\right)}\right)\right) \\
=\sum_{R \vdash(m+n)} g(r, s, R) \operatorname{Dim}_{N} R\left(-\sum_{\square \in R} c_{\square}(R)+\sum_{\square \in r} c_{\square}(r)+\sum_{\square \in s} c_{\square}(s)\right)
\end{array}
$$

Comparing (3.10) and (3.11), we have

$$
\frac{(m+n) !}{(m-1) !(n-1) !}=N \sum_{R \vdash(m+n)} g(r, s, R) \frac{d_{R}}{d_{r} d_{s}} \frac{f_{R}}{f_{r} f_{s}}\left(-\sum_{\square \in R} c_{\square}(R)+\sum_{\square \in r} c_{\square}(r)+\sum_{\square \in s} c_{\square}(s)\right)
$$

Consider the large $N$ expansion.

$$
\frac{f_{R}}{f_{r} f_{s}}=1+\frac{1}{N}\left(-\sum_{\square \in R} c_{\square}(R)+\sum_{\square \in r} c_{\square}(r)+\sum_{\square \in s} c_{\square}(s)\right)+\mathcal{O}\left(\frac{1}{N^{2}}\right)
$$

Fraction $\frac{f_{R}}{f_{r} f_{s}}$ is the only term in the summand of the r.h.s. in (3.12) which contains $N$ dependence. Considering the order $N$ term of the r.h.s., we get zero using the first identity in (3.6). Considering the constant term, we get

$$
\frac{(m+n) !}{(m-1) !(n-1) !}=\sum_{R \vdash(m+n)} g(r, s, R) \frac{d_{R}}{d_{r} d_{s}}\left(-\sum_{\square \in R} c_{\square}(R)+\sum_{\square \in r} c_{\square}(r)+\sum_{\square \in s} c_{\square}(s)\right)^{2} .
$$

Equivalently,

$$
\frac{(m+n) !}{(m-1) !(n-1) !} d_{r} d_{s}=\sum_{R \vdash(m+n)} g(r, s, R) d_{R}\left(-\sum_{\square \in R} c_{\square}(R)+\sum_{\square \in r} c_{\square}(r)+\sum_{\square \in s} c_{\square}(s)\right)^{2} .
$$

Now it is easy to see that this is a generalization of (2.22). 


\section{Multi-partite generalization}

Consider $S_{n_{1}} \times \ldots \times S_{n_{k}} \rightarrow S_{n_{1}+\ldots+n_{k}}$ and tensor space $W=V_{N}^{\otimes n_{1}+\ldots+n_{k}}$.

For Young diagrams $r_{1}, r_{2}, \cdots, r_{k}$ with $n_{1}, n_{2}, \cdots, n_{k}$ boxes, we have representations $V_{r_{1}}^{U}, V_{r_{2}}^{U}, \cdots, V_{r_{k}}^{U}$ of $\mathrm{U}(N)$. Considering the decomposition of the tensor product under the diagonal action of $\mathrm{U}(N)$, we have

$$
V_{r_{1}}^{U} \otimes V_{r_{2}}^{U} \otimes \cdots \otimes V_{r_{k}}^{U}=\bigoplus_{R \vdash n_{1}+\cdots+n_{k}} g\left(r_{1}, r_{2}, \cdots, r_{k} ; R\right) V_{R}^{U}
$$

The multiplicities $g\left(r_{1}, \cdots, r_{k} ; R\right)$ can be expressed in terms of Littlewood-Richardson coefficients. For example

$$
g\left(r_{1}, r_{2}, r_{3} ; R\right)=\sum_{S \vdash n_{1}+n_{2}} g\left(r_{1}, r_{2} ; S\right) g\left(S, r_{3} ; R\right)
$$

By considering the dimensions on the two sides of (4.1), we have

$$
\operatorname{Dim}_{N} r_{1} \ldots \operatorname{Dim}_{N} r_{k}=\sum_{R \vdash\left(n_{1}+\ldots+n_{k}\right)} g\left(r_{1}, \ldots, r_{k} ; R\right) \operatorname{Dim}_{N} R
$$

Using (2.6) the above equation can be rewritten as

$$
\frac{\left(n_{1}+\ldots+n_{k}\right) !}{n_{1} ! \ldots n_{k} !} d_{r_{1}} \ldots d_{r_{k}}=\sum_{R \vdash\left(n_{1}+\ldots+n_{k}\right)} g\left(r_{1}, \ldots, r_{k} ; R\right) d_{R} \frac{f_{R}}{f_{r_{1}} \ldots f_{r_{k}}}
$$

Now we switch to considering the induction of representations of symmetric groups associated with the above Young diagrams. Using induction of $V_{r_{1}}^{S_{n_{1}}} \otimes \ldots \otimes V_{r_{k}}^{S_{n_{k}}}$ from $S_{n_{1}} \times \ldots \times S_{n_{k}}$ to $S_{n_{1}+\ldots+n_{k}}$ we have

$$
\frac{\left(n_{1}+\ldots+n_{k}\right) !}{n_{1} ! \ldots n_{k} !} d_{r_{1}} \ldots d_{r_{k}}=\sum_{R} g\left(r_{1}, \ldots, r_{k} ; R\right) d_{R}
$$

Comparing equations (4.4), (4.5) we have

$$
0=\sum_{R \vdash\left(n_{1}+\ldots+n_{k}\right)} g\left(r_{1}, \ldots, r_{k} ; R\right) d_{R}\left(1-\frac{f_{R}}{f_{r_{1}} \ldots f_{r_{k}}}\right)
$$

Again we consider a large $N$ expansion of

$$
\frac{f_{R}}{f_{r_{1}} \ldots f_{r_{k}}}=\frac{\prod_{\square \in R}\left(1-\frac{c_{\square}(R)}{N}\right)}{\prod_{\square \in r_{1}}\left(1-\frac{c_{\square}\left(r_{1}\right)}{N}\right) \ldots \prod_{\square \in r_{k}}\left(1-\frac{c_{\square}\left(r_{k}\right)}{N}\right)}
$$

Validity of eq. (4.6) for all $N \geq \sum_{j=1}^{k} n_{j}$ leads to identities for every power of $1 / N$. For example, the $1 / N$ terms give

$$
0=\sum_{R \vdash\left(n_{1}+\ldots+n_{k}\right)} g\left(r_{1}, \ldots, r_{k}, R\right) d_{R}\left(-\sum_{\square \in R} c_{\square}(R)+\sum_{i=1}^{k} \sum_{\square \in r_{i}} c_{\square}\left(r_{i}\right)\right)
$$


It is useful to be explicit about the embedding of $S_{n_{1}} \times \cdots S_{n_{k}}$ in $S_{n_{1}+\cdots n_{k}}$. Let $S_{n_{1}}$ be the group of permutations of $\left[n_{1}\right]=\left\{1,2, \cdots, n_{1}\right\}$. Let $S_{n_{2}}$ be the group of permutations of $\left[n_{2}\right]=\left\{n_{1}+1, \cdots, n_{1}+n_{2}\right\}$. And $S_{n_{i}}$ for $1 \leq i \leq k$ be the group of permutations of

$$
\left[n_{i}\right]=\left\{n_{1}+n_{2}+\cdots n_{i-1}+1, \cdots, n_{1}+n_{2}+\cdots+n_{i}\right\}
$$

We also let $S_{n_{1}+n_{2}+\cdots n_{1}+n_{2}+\cdots n_{k}}$ be the group of permutations of $\left\{1,2, \cdots, n_{1}+n_{2}+\cdots+n_{k}\right\}$. Let us evaluate the trace

$$
\operatorname{tr}_{W}\left(\left(\frac{P_{r_{1}}}{d_{r_{1}}} \otimes \ldots \otimes \frac{P_{r_{k}}}{d_{r_{k}}}\right)\left(T_{2}^{\left(S_{n_{1}+\ldots+n_{k}}\right)}-\sum_{i=1}^{k} T_{2}^{\left(S_{n_{i}}\right)}\right)\right)
$$

in two ways. We observe that

$$
T_{2}^{\left(S_{n_{1}+\ldots+n_{k}}\right)}-\sum_{i=1}^{k} T_{2}^{\left(S_{n_{i}}\right)}=\sum_{i=1}^{k-1} \sum_{j=i+1}^{k} \sum_{a \in\left[n_{i}\right]} \sum_{b \in\left[n_{j}\right]}(a, b) .
$$

Direct calculation (analogous to eq. (3.11)) gives

$$
\begin{aligned}
& \operatorname{tr}_{W}\left(\left(\frac{P_{r_{1}}}{d_{r_{1}}} \otimes \ldots \otimes \frac{P_{n_{k}}}{d_{n_{k}}}\right)\left(T_{2}^{\left(S_{n_{1}+\ldots+n_{k}}\right)}-\sum_{i=1}^{k} T_{2}^{\left(S_{n_{i}}\right)}\right)\right) \\
& =\frac{1}{n_{1} ! \ldots n_{k} !} \sum_{\sigma_{1} \in S_{n_{1}}} \ldots \sum_{\sigma_{k} \in S_{n_{k}}} \\
& \quad \times \chi_{r_{1}}\left(\sigma_{1}\right) \ldots \chi_{r_{k}}\left(\sigma_{k}\right) \operatorname{tr}_{W}\left(\left(\sigma_{1} \otimes \ldots \otimes \sigma_{k}\right) \sum_{i=1}^{k-1} \sum_{j=i+1}^{k} \sum_{a \in\left[n_{i}\right]} \sum_{b \in\left[n_{j}\right]}(a, b)\right) \\
& =\frac{\sum_{i=1}^{k-1} \sum_{j=i+1}^{k} n_{i} n_{j}}{n_{1} ! \ldots n_{k} !} \sum_{\sigma_{1} \in S_{n_{1}}} \ldots \sum_{\sigma_{k} \in S_{n_{k}}} \chi_{r_{1}}\left(\sigma_{1}\right) \ldots \chi_{r_{k}}\left(\sigma_{k}\right) N^{C_{\sigma_{1}}+\ldots+C_{\sigma_{k}}-1} \\
& =\frac{\sum_{i=1}^{k-1} \sum_{j=i+1}^{k} n_{i} n_{j}}{N} \operatorname{Dim}_{N} r_{1} \ldots \operatorname{Dim}_{N} r_{k}
\end{aligned}
$$

On the other hand, using (A.11), (A.12) and (A.27), we have

$$
\begin{aligned}
& \operatorname{tr}_{W}\left(\left(\frac{P_{r_{1}}}{d_{r_{1}}} \otimes \ldots \otimes \frac{P_{n_{k}}}{d_{n_{k}}}\right)\left(T_{2}^{\left(S_{n_{1}+\ldots+n_{k}}\right)}-\sum_{i=1}^{k} T_{2}^{\left(S_{n_{i}}\right)}\right)\right) \\
& =\sum_{R \vdash\left(n_{1}+\ldots+n_{k}\right)} g\left(r_{1}, \ldots, r_{k}, R\right) \operatorname{Dim}_{N} R\left(-\sum_{\square \in R} c_{\square}(R)+\sum_{i=1}^{k} \sum_{\square \in r_{i}} c_{\square}\left(r_{i}\right)\right)
\end{aligned}
$$

Comparing (4.12) and (4.13), we have

$$
\begin{aligned}
& \left(\sum_{i=1}^{k-1} \sum_{j=i+1}^{k} n_{i} n_{j}\right) \frac{\left(n_{1}+\ldots+n_{k}\right) !}{n_{1} ! \ldots n_{k} !} \\
& =N \sum_{R \vdash\left(n_{1}+\ldots+n_{k}\right)} g\left(r_{1}, \ldots, r_{k}, R\right) \frac{d_{R}}{d_{r_{1}} \ldots d_{r_{k}}} \frac{f_{R}}{f_{r_{1}} \ldots f_{r_{k}}}\left(-\sum_{\square \in R} c_{\square}(R)+\sum_{i=1}^{k} \sum_{\square \in r_{i}} c_{\square}\left(r_{i}\right)\right)
\end{aligned}
$$


Consider the large $N$ expansion.

$$
\frac{f_{R}}{f_{r_{1}} \ldots f_{r_{k}}}=1+\frac{1}{N}\left(-\sum_{\square \in R} c_{\square}(R)+\sum_{i=1}^{k} \sum_{\square \in r_{i}} c_{\square}\left(r_{i}\right)\right)+\mathcal{O}\left(\frac{1}{N^{2}}\right)
$$

In eq. (4.14) the only term in the summand of the r.h.s., which contains $N$ dependence is the fraction $\frac{f_{R}}{f_{r_{1} \ldots} \ldots f_{r_{k}}}$. Considering the order $N$ term of the r.h.s., we get zero using the first identity in (4.8). Considering the constant term, we get

$$
\begin{aligned}
& \left(\sum_{i=1}^{k-1} \sum_{j=i+1}^{k} n_{i} n_{j}\right) \frac{\left(n_{1}+\ldots+n_{k}\right) !}{n_{1} ! \ldots n_{k} !} \\
& =\sum_{R \vdash\left(n_{1}+\ldots+n_{k}\right)} g\left(r_{1}, \ldots, r_{k}, R\right) \frac{d_{R}}{d_{r_{1}} \ldots d_{r_{k}}}\left(-\sum_{\square \in R} c_{\square}(R)+\sum_{i=1}^{k} \sum_{\square \in r_{i}} c_{\square}\left(r_{i}\right)\right)^{2}
\end{aligned}
$$

This can be equivalently rewritten as

$$
\begin{aligned}
& \left(\sum_{i=1}^{k-1} \sum_{j=i+1}^{k} n_{i} n_{j}\right) \frac{\left(n_{1}+\ldots+n_{k}\right) !}{n_{1} ! \ldots n_{k} !} d_{r_{1}} \ldots d_{r_{k}} \\
& =\sum_{R \vdash\left(n_{1}+\ldots+n_{k}\right)} g\left(r_{1}, \ldots, r_{k}, R\right) d_{R}\left(-\sum_{\square \in R} c_{\square}(R)+\sum_{i=1}^{k} \sum_{\square \in r_{i}} c_{\square}\left(r_{i}\right)\right)^{2}
\end{aligned}
$$

Wee see that this is a generalization of (3.15).

\section{Permutation centralizer algebras, composite gauge invariant operators and AdS/CFT}

The identities above have been derived by calculating the trace in tensor spaces of some elements in the group algebra of $S_{n_{1}+n_{2}+\cdots+n_{k}}$, which are invariant under conjugation of by permutations in $S_{n_{1}} \times S_{n_{2}} \times \cdots S_{n_{k}}$. Let us specialise to the case $k=2$. The subspace of $\mathbb{C}\left(S_{m+n}\right)$ which is invariant under conjugation by $S_{m} \times S_{n}$ forms an algebra which has been studied in detail in $[4,5]$. The motivation came from the role these played in the construction of bases of gauge invariant operators which diagonalise an inner product coming from free quantum field theory [6-10]. Key insights into the construction of these bases came from the physics of strings attached to branes in the context of the AdS/CFT correspondence [11-13].

Consider quantum fields $X, Y$ which are $N \times N$ matrices transforming in the adjoint of a $\mathrm{U}(N)$ gauge symmetry.

$$
\begin{aligned}
& X \rightarrow U X U^{\dagger} \\
& Y \rightarrow U Y U^{\dagger}
\end{aligned}
$$

For large $N$, the space of gauge invariant operators is in 1-1 correspondence with the elements of $\mathcal{A}(m, n)$. One way to count the dimension of this space is to count the traces, 
which amounts to counting cyclic words built from two letters. As explained in the references above (and reviewed in [14]) the dimension of the space of gauge invariant operators is also given in terms of Littlewood-Richardson coefficients $g\left(R_{1}, R_{2}, R_{3}\right)$ which are multiplicities for the $\mathrm{U}(N)$ representation associated with Young diagram $R_{3}$ (having $m+n$ boxes) to appear in the tensor product of $R_{1} \otimes R_{2}$, where $R_{1}$ and $R_{2}$ have $m$ and $n$ boxes.

$$
\operatorname{Dim}(\mathcal{A}(m, n))=\sum_{\substack{R_{1} \vdash m \\ R_{2} \vdash n}} \sum_{R_{3} \vdash m+n}\left(g\left(R_{1}, R_{2}, R_{3}\right)\right)^{2}
$$

The finite $N$ counting is given simply by restricting $R_{3}$ to have no more than $N$ rows. This follows by application of Schur-Weyl duality. The reason these permutation equivalences arise in constructing gauge invariants is that if we consider a general operator

$$
X_{j_{1}}^{i_{1}} \cdots X_{j_{n}}^{i_{n}} Y_{j_{n+1}}^{i_{n+1}} \cdots Y_{j_{n+m}}^{i_{n+m}}
$$

the upper indices transform in $V^{\otimes(m+n)}$ of $\mathrm{U}(N)$. The lower indices transform as $\bar{V}^{\otimes(m+n)}$. The invariants of $\mathrm{U}(N)$ are obtained by contracting with Kronecker $\delta$ 's. As a result, we can construct a gauge invariant for every permutation $\sigma \in S_{m+n}$.

$$
\mathcal{O}_{\sigma}(X, Y)=X_{i_{\sigma(1)}}^{i_{1}} \cdots X_{i_{\sigma(n)}}^{i_{n}} Y_{i_{\sigma(n+1)}}^{i_{n+1}} \cdots Y_{i_{\sigma(n+m)}}^{i_{n+m}}
$$

The bosonic symmetry leads to an equivalence

$$
\mathcal{O}_{\sigma}(X, Y)=\mathcal{O}_{\gamma \sigma \gamma^{-1}}(X, Y)
$$

for all $\gamma \in S_{m} \times S_{n}$. Fourier transformation on $\mathcal{A}(m, n)$ using representation theory of symmetric groups leads to a Young diagram basis $Q_{R_{1}, R_{2} ; \nu_{1}, \nu_{2}}^{R_{3}}$, with $1 \leq \nu_{1}, \nu_{2} \leq g\left(R_{1}, R_{2}, R_{3}\right)$. Representation theoretic formulae for the Fourier coefficients giving the transformation from trace basis to the Young diagram basis are given in the papers above. Structural questions about $\mathcal{A}(m, n)$, notably regarding minimal sets of generators for maximal commuting sub-algebras, are related to the question of how many charges (generalized Casimirs) are needed to specify a state in the 2 -matrix system $[4,15]$. This can be considered to be a measure of complexity of this state space.

$\mathcal{A}(m, n)$ is an example of a permutation centralizer algebra. $\mathcal{A}\left(n_{1}, \cdots, n_{k}\right)$ is analogously defined, and is relevant to gauge invariant operators made from $k$ flavours of matrix quantum fields. The elements we have used to get the identities above are in fact special elements which are central in $\mathcal{A}\left(n_{1}, \cdots, n_{k}\right)$. The central subspace is spanned by products of elements from the centre of $\mathbb{C}\left(S_{\sum_{i} n_{i}}\right)$ with elements from the centre of $\prod_{i} \mathbb{C}\left(S_{n_{i}}\right)$. (these properties of the centre of PCAs are explained in [4] and the special role of the centre in terms of the complexity of correlator computations is discussed). The traces of central elements can thus be obtained using character formulae for symmetric groups [16].

In fact any central element of $\mathcal{A}\left(n_{1}, \cdots, n_{k}\right)$ will lead to an identity of the kind we discussed in the earlier sections.

We may make a few remarks about the analogies which are emerging between quantum information processing and gauge invariant composite quantum fields through the shared 
feature of permutation centralizer algebras and Schur-Weyl duality. In QIP, multiple uses of a unitary operation occur in the computational tasks like oracle based algorithms, estimation problems or arbitrary protocols, which should perform equally well for all states or channels. ${ }^{1}$ In QFT, these multiple uses of a unitary $U$ arise through the action on a polynomial composite quantum field.

We thus have a first simple interesting analogy, somewhat simplified from the above set-up, which seems to hold promise of wider implications:

A unitary quantum channel is analogous to a unitary gauge transformation of an elementary quantum field.

In the QIP problem, multiple uses of channels occur within multi step quantum protocols (i.e. within networks of quantum channels). In the composite operator problem of QFT, multiple uses occur in different copies of the elementary quantum field occuring within a composite. We thus have a second simplified analogy to think about.

A multi step quantum protocol is analogous to a composite local operator.

The simplicity of these analogies seems to suggest there should be wider applications. For example, for the multi-partite generalization in section 4 we may ask, is there an appropriate optimization task in quantum information theory involving multiple quantum devices interacting with each other in some way, which employs the multi-partitite identities (4.17) - generalizing the use of (2.22) in perfect probabilistic storage and retrieval [1]?

As noted earlier in this section, structural questions about PCAs have been used to characterize the complexity of quantum states in multi-matrix systems, which have a Young diagram basis as well as a trace basis. The Schur-Weyl duality transformation from tensor product basis to the Young diagram basis for $V_{N}^{\otimes n}$ has been studied from a quantum information perspective [22]. The question of efficient quantum circuits having polynomial number of gates has been addressed. Similar questions can be studied for the transformation from trace basis to Young diagram basis for multi-matrix systems. The definition of complexity of quantum circuits requires a choice of a basic gate set. A reasonable choice in the context of AdS/CFT would be to consider the quantum dilatation operator at one loop and higher loops (see [23] for the 1-loop dilatation operator and [24, 25] for applications to brane physics of the action of the one-loop dilatation operator on the Young diagram basis). A challenge would be to identify an AdS/CFT dual for such a notion of circuit complexity involving the quantum dilatation operator in the 2-matrix system.

As we have seen, permutation centralizer algebras, with their traces illuminating aspects of perfect probabilistic storing/retrieving and their structure constants having information about correlators of relevance to AdS/CFT, provide an intriguing mathematical connection between quantum information and AdS/CFT. An interesting question is whether there is a physical interpretation of this mathematical connection between QIP and AdS/CFT. In this connection, it is worth noting that studies of quantum state spaces

\footnotetext{
${ }^{1}$ For a broad discussion of group techniques in quantum information tasks, see [17]. For use of symmetries in multi step quantum protocols see [18-21].
} 
in AdS/CFT from information theoretic perspectives have been undertaken [26-28], primarily in the context of state spaces associated with invariants of a single matrix and the related free fermion system. More broadly on this theme the work of [29] has motivated a rich exploration of connections between AdS/CFT and quantum information. For example it has led to the idea of space-time emerging from entanglement [30] with implications for AdS/CFT holography [31] and black hole physics [32].

\section{Acknowledgments}

SR is supported by the STFC consolidated grant ST/L000415/1 "String Theory, Gauge Theory \& Duality" and a Visiting Professorship at the University of the Witwatersrand, funded by a Simons Foundation grant to the Mandelstam Institute for Theoretical Physics. SR thanks the Galileo Galilei Institute for Theoretical Physics for hospitality and the INFN for partial support during the completion of this work. He also thanks the organizers of the workshop on Matrix Models for non-commutative geometry and string theory in Vienna and the KEK theory group for hospitality during the completion of this project. We are grateful for useful discussions to David Berenstein, Robert de Mello Koch, Costis Papageorgakis, Rodolfo Russo, Masaki Shigemori. MS acknowledges the support by the QuantERA project HIPHOP (project ID 731473), projects QETWORK (APVV-14-0878), MAXAP (VEGA 2/0173/17), GRUPIK (MUNI/G/1211/2017) and GAČR No. GA1622211S. MS is grateful to A. Bisio and M. Ziman for fruitful discussions and collaborative work, which led to formulation of the identity (1.1), which is re-derived and generalized in this manuscript. We are grateful to the organizers of the Quantum Physics and Logic (QPL2017) conference where this interdisciplinary collaboration was initiated.

\section{A some facts about $\mathrm{U}(N), S_{n}$ and the tensor product $V_{N}^{\otimes n}$}

This section is brief review of some key facts about the representation theory of symmetric groups, Unitary groups and their relations following from Schur-Weyl duality. More details are in mathematical physics references such as [33] or mathematics texts such as [3]. We will start with a useful piece of notation. We will use $r \vdash n$ to denote a partition $r$ of $n$. Partitions of $n$ correspond to Young diagrams with $n$ boxes, which have row lengths $r_{1} \geq r_{2} \geq \cdots$, with $n=r_{1}+r_{2}+\cdots$. Young diagrams with $n$ boxes correspond to irreducible representations of $S_{n}$. Letting $V_{N}$ be the fundamental representation of $\mathrm{U}(N)$, the tensor product $V_{N}^{\otimes n}$ is a representation of the diagonal $\mathrm{U}(N)$ acting as

$$
U \otimes U \otimes \cdots \otimes U
$$

as well as the symmetric group of all permutations of $n$ objects $\left(S_{n}\right)$. These two actions commute with each other, which leads to Schur-Weyl duality

$$
V_{N}^{\otimes n}=\bigoplus_{r \vdash n} V_{r}^{\mathrm{U}(N)} \otimes V_{r}^{S_{n}}
$$


This gives the decomposition of $V_{N}^{\otimes n}$ into irreducible reps of $\mathrm{U}(N) \times S_{n}$ as a direct sum labelled by Young diagrams.

A useful formula for the dimension of unitary group $\mathrm{U}(N)$ irreps in terms of characters of $S_{n}$ is

$$
\frac{1}{n !} \sum_{\sigma \in S_{n}} \chi_{r}(\sigma) N^{C_{\sigma}}=\operatorname{Dim}_{N} r
$$

where $C_{\sigma}$ is the number of cycles in the permutation $\sigma$. This follows from Schur-Weyl duality (A.2). To project to a fixed Young diagram, we can use a projector element in the group algebra

$$
P_{r}=\frac{d_{r}}{n !} \sum_{\sigma \in S_{n}} \chi_{r}(\sigma) \sigma
$$

If we apply this to the states in $V_{N}^{\otimes n}$ and take a trace, we need to calculate

$$
\begin{aligned}
\operatorname{tr}_{V_{N}^{\otimes n}}(\sigma) & =\left\langle e_{i_{1}} \otimes \cdots \otimes e_{i_{n}}|\sigma| e_{i_{1}} \otimes \cdots \otimes e_{i_{n}}\right\rangle \\
& =\left\langle e_{i_{1}} \otimes \cdots \otimes e_{i_{n}} \mid e_{i_{\sigma(1)}} \otimes \cdots \otimes e_{i_{\sigma(n)}}\right\rangle \\
& =N^{C_{\sigma}}
\end{aligned}
$$

( usual summation convention, so the $i$ indices are summed from 1 to $N$ ). To understand the last line, it is instructive to do some examples at $n=2$. If $\sigma=(1)(2)$, the trace is

$$
\delta_{i_{1}, i_{1}} \delta_{i_{2}, i_{2}}=N^{2}
$$

If $\sigma=(12)$, the trace is

$$
\delta_{i_{1}, i_{\sigma(1)}} \delta_{i_{2}, i_{\sigma(2)}}=\delta_{i_{1}, i_{2}} \delta_{i_{2}, i_{1}}=N
$$

We need to understand some multiplications in the group algebra of $S_{n+1}$. The group algebra consists of formal sums of group elements with complex coefficients. What happens when a generic group element $\sigma$ in the $S_{n}$ subgroup is multiplied with $(a, n+1)$ for $a \in$ $\{1, \cdots, n\}$ ? Example at $n=3$, with $\sigma=(1,2,3)$

$$
(1,2,3)(2,4)=(1,4,2,3)
$$

The number of cycles in $\sigma \cdot(a, n+1)$ is the same as the number of cycles in $\sigma$. As a result, if

$$
\begin{aligned}
\tau & =\sigma \cdot \sum_{a=1}^{n}(a, n+1) \\
C_{\tau} & =C_{\sigma}
\end{aligned}
$$

This implies that

$$
\begin{aligned}
\operatorname{tr}_{V_{N}^{\otimes n+1}}\left(\frac{P_{r}}{d_{r}} \sum_{a=1}^{n}(a, n+1)\right) & =\frac{1}{n !} \sum_{a=1}^{n} \sum_{\sigma \in S_{n}} \chi_{r}(\sigma) \operatorname{tr}_{V_{N}^{\otimes n+1}}(\sigma .(a, n+1)) \\
& =\sum_{a=1}^{n} \operatorname{Dim}_{N} r=n \operatorname{Dim}_{N} r
\end{aligned}
$$


Central elements ( $\operatorname{such}$ as $T_{2}^{\left(S_{n}\right)}$ ) multiplying a projector give normalized characters times the projector.

$$
T_{2}^{\left(S_{n}\right)} P_{r}=\frac{\chi_{r}\left(T_{2}^{S_{n}}\right)}{d_{r}} P_{r}
$$

To see this, note that both l.h.s. and r.h.s. are central elements in the group algebra of $S_{n}$, as a result they are determined by their irreducible characters, and we can easily verify that the two sides have the same irreducible characters. The normalized character is known [16] to be the sum of contents

$$
\frac{\chi_{r}\left(T_{2}^{S_{n}}\right)}{d_{r}}=\sum_{\square \in r}\left(-c_{\square}(r)\right)
$$

An implication of Schur-Weyl duality is that the Littlewood-Richardson coefficients $g\left(r_{1}, r_{2} ; R\right)$ which give the multiplicities of $\mathrm{U}(N)$ tensor product decompositions

$$
V_{r_{1}}^{U} \otimes V_{r_{2}}^{U}=\oplus_{R} g\left(r_{1}, r_{2}, R\right) V_{R}^{U}
$$

also have an interpretation purely in terms of symmetric groups. They are the reduction multiplicities for the decomposition of the irrep $V_{R}^{S_{n}}$ in terms of the subgroup $S_{n_{1}} \times S_{n_{2}}$. We may express this as

$$
V_{R}^{S_{n}}=\bigoplus_{r_{1}, r_{2}} V_{r_{1}}^{S_{n_{1}}} \otimes V_{r_{2}}^{S_{n_{2}}} \otimes V_{r_{1}, r_{2}}^{R}
$$

where $V_{r_{1}, r_{2}}^{R}$ is the multiplicity space, of dimension $g\left(r_{1}, r_{2}, R\right)$. Considering the trace in $V_{R}^{S_{n}}$

$$
\operatorname{tr}\left(P_{r_{1}} \circ P_{r_{2}}\right)
$$

we arrive at

$$
g\left(r_{1}, r_{2}, R\right)=\frac{1}{n_{1} ! n_{2} !} \sum_{\sigma_{1} \in S_{n_{1}}} \sum_{\sigma_{2} \in S_{n_{2}}} \chi_{r_{1}}\left(\sigma_{1}\right) \chi_{r_{2}}\left(\sigma_{2}\right) \chi_{R}\left(\sigma_{1} \circ \sigma_{2}\right)
$$

More generally

$$
V_{r_{1}}^{U} \otimes V_{r_{2}}^{U} \otimes \cdots \otimes V_{r_{k}}^{U}=\oplus_{R} \quad g\left(r_{1}, r_{2}, \cdots r_{k} ; R\right) V_{R}^{U}
$$

and

$$
g\left(r_{1}, r_{2}, \cdots r_{k} ; R\right)=\sum_{\sigma_{1} \in S_{n_{1}}} \cdots \sum_{\sigma_{k} \in S_{n_{k}}}\left(\prod_{i=1}^{k} \frac{\chi_{r_{i}}\left(\sigma_{i}\right)}{n_{i} !}\right) \chi_{R}\left(\sigma_{1} \circ \sigma_{2} \circ \cdots \circ \sigma_{k}\right)
$$

Let $W=V_{N}^{\otimes n}=V_{N}^{\otimes\left(n_{1}+n_{2}+\cdots+n_{k}\right)}$. Consider the trace

$$
\operatorname{tr}_{W} P_{R}\left(\frac{P_{r_{1}}}{d_{r_{1}}} \otimes \frac{P_{r_{2}}}{d_{r_{2}}} \otimes \cdots \otimes \frac{P_{r_{k}}}{d_{r_{k}}}\right)
$$


where $R$ is a Young diagram with $n$ boxes, $r_{i}$ are Young diagrams with $n_{i}$ boxes, $P_{R}$ and $P_{r_{i}}$ are the corresponding projectors.Using Schur-Weyl duality

$$
W=V_{N}^{\otimes n}=\bigoplus_{R \vdash n} V_{R}^{U} \otimes V_{R}^{S_{n}}
$$

When the projector $P_{R}$ acts on $W$, we project to a single factor $V_{R}^{U} \otimes V_{R}^{S_{n}}$. We can decompose $V_{R}^{S_{n}}$ in terms of $S_{n_{1}} \times S_{n_{2}} \times \cdots \times S_{n_{k}}$. The multiplicities are the LittlewoodRichardson coefficients.

$$
V_{R}^{U} \otimes V_{R}^{S_{n}}=V_{R}^{U} \otimes \bigoplus_{r_{1}, r_{2}, \cdots, r_{k}} g\left(r_{1}, r_{2}, \cdots, r_{k} ; R\right)\left(V_{r_{1}}^{S_{n_{1}}} \otimes V_{r_{2}}^{S_{n_{2}}} \otimes \cdots \otimes V_{r_{k}}^{S_{n_{k}}}\right)
$$

It follows that

$$
\operatorname{tr}_{W} P_{R}\left(\frac{P_{r_{1}}}{d_{r_{1}}} \otimes \frac{P_{r_{2}}}{d_{r_{2}}} \otimes \cdots \otimes \frac{P_{r_{k}}}{d_{r_{k}}}\right)=\left(\operatorname{Dim}_{N} R\right) g\left(r_{1}, r_{2}, \cdots, r_{k} ; R\right)
$$

This is an important identity we use in the paper. To make the above proof more explicit, we can expand the projectors in terms of characters.

$$
\begin{aligned}
& \operatorname{tr}_{W} P_{R}\left(\frac{P_{r_{1}}}{d_{r_{1}}} \otimes \frac{P_{r_{2}}}{d_{r_{2}}} \otimes \cdots \otimes \frac{P_{r_{k}}}{d_{r_{k}}}\right) \\
& =\sum_{\sigma_{1} \in S_{n_{1}}} \cdots \sum_{\sigma_{k} \in S_{n_{k}}} \sum_{\sigma \in S_{n}} \frac{d_{R} \chi_{R}(\sigma)}{n !}\left(\prod_{i=1}^{k} \frac{\chi_{r_{i}}\left(\sigma_{i}\right)}{n_{i} !}\right) \operatorname{tr}_{W}\left(\sigma\left(\sigma_{1} \circ \sigma_{2} \circ \cdots \circ \sigma_{k}\right)\right)
\end{aligned}
$$

For any permutation $\tau \in S_{n}$, Schur-Weyl duality implies that

$$
\operatorname{tr}_{W}(\tau)=\sum_{S \vdash n} \chi_{S}(\tau) \operatorname{Dim}_{N} S
$$

Hence

$$
\begin{aligned}
& \operatorname{tr}_{W} P_{R}\left(\frac{P_{r_{1}}}{d_{r_{1}}} \otimes \frac{P_{r_{2}}}{d_{r_{2}}} \otimes \cdots \otimes \frac{P_{r_{k}}}{d_{r_{k}}}\right) \\
& =\sum_{\sigma_{1} \in S_{n_{1}}} \cdots \sum_{\sigma_{k} \in S_{n_{k}}} \sum_{\sigma \in S_{n}} \frac{d_{R} \chi_{R}(\sigma)}{n !}\left(\prod_{i=1}^{k} \frac{\chi_{r_{i}}\left(\sigma_{i}\right)}{n_{i} !}\right) \sum_{S \vdash n} \chi_{S}\left(\sigma\left(\sigma_{1} \circ \sigma_{2} \circ \cdots \circ \sigma_{k}\right)\right) \operatorname{Dim}_{N} S
\end{aligned}
$$

Using the character orthogonality relation

$$
\frac{1}{n !} \sum_{\sigma} \chi_{S}(\sigma) \chi_{R}(\sigma \rho)=\delta_{R S} \frac{\chi_{R}(\rho)}{d_{R}}
$$

which holds for any $\rho \in S_{n}$, we have

$$
\begin{aligned}
& \operatorname{tr}_{W} P_{R}\left(\frac{P_{r_{1}}}{d_{r_{1}}} \otimes \frac{P_{r_{2}}}{d_{r_{2}}} \otimes \cdots \otimes \frac{P_{r_{k}}}{d_{r_{k}}}\right) \\
& =\sum_{\sigma_{1} \in S_{n_{1}}} \cdots \sum_{\sigma_{k} \in S_{n_{k}}} \sum_{\sigma \in S_{n}}\left(\prod_{i=1}^{k} \frac{\chi_{r_{i}}\left(\sigma_{i}\right)}{n_{i} !}\right) \chi_{R}\left(\sigma_{1} \circ \sigma_{2} \circ \cdots \circ \sigma_{k}\right) \operatorname{Dim}_{N} R \\
& =g\left(r_{1}, r_{2}, \cdots, r_{k} ; R\right) \operatorname{Dim}_{N} R
\end{aligned}
$$


Open Access. This article is distributed under the terms of the Creative Commons Attribution License (CC-BY 4.0), which permits any use, distribution and reproduction in any medium, provided the original author(s) and source are credited.

\section{References}

[1] M. Sedlák, A. Bisio and M. Ziman, Optimal probabilistic storage and retrieval of unitary channels, arXiv:1809.04552.

[2] M. Sedlák and A. Bisio, On some new hook content identities, arXiv:1809.02008.

[3] W. Fulton and J. Harris, Representation Theory: a first course, Springer (2004).

[4] P. Mattioli and S. Ramgoolam, Permutation Centralizer Algebras and Multi-Matrix Invariants, Phys. Rev. D 93 (2016) 065040 [arXiv:1601.06086] [INSPIRE].

[5] Y. Kimura, Noncommutative Frobenius algebras and open-closed duality, arXiv:1701.08382 [INSPIRE].

[6] Y. Kimura and S. Ramgoolam, Branes, anti-branes and brauer algebras in gauge-gravity duality, JHEP 11 (2007) 078 [arXiv:0709.2158] [INSPIRE].

[7] T.W. Brown, P.J. Heslop and S. Ramgoolam, Diagonal multi-matrix correlators and BPS operators in $N=4 S Y M$, JHEP 02 (2008) 030 [arXiv:0711.0176] [INSPIRE].

[8] R. Bhattacharyya, S. Collins and R. de Mello Koch, Exact Multi-Matrix Correlators, JHEP 03 (2008) 044 [arXiv: 0801.2061] [INSPIRE].

[9] R. Bhattacharyya, R. de Mello Koch and M. Stephanou, Exact Multi-Restricted Schur Polynomial Correlators, JHEP 06 (2008) 101 [arXiv:0805.3025] [INSPIRE].

[10] T.W. Brown, P.J. Heslop and S. Ramgoolam, Diagonal free field matrix correlators, global symmetries and giant gravitons, JHEP 04 (2009) 089 [arXiv:0806.1911] [INSPIRE].

[11] S. Corley, A. Jevicki and S. Ramgoolam, Exact correlators of giant gravitons from dual $N=4$ SYM theory, Adv. Theor. Math. Phys. 5 (2002) 809 [hep-th/0111222] [INSPIRE].

[12] V. Balasubramanian, M.-x. Huang, T.S. Levi and A. Naqvi, Open strings from $N=4$ superYang-Mills, JHEP 08 (2002) 037 [hep-th/0204196] [INSPIRE].

[13] V. Balasubramanian, D. Berenstein, B. Feng and M.-x. Huang, D-branes in Yang-Mills theory and emergent gauge symmetry, JHEP 03 (2005) 006 [hep-th/0411205] [INSPIRE].

[14] S. Ramgoolam, Permutations and the combinatorics of gauge invariants for general $N$, PoS (CORFU2015) 107 (2016) [arXiv: 1605. 00843] [INSPIRE].

[15] Y. Kimura and S. Ramgoolam, Enhanced symmetries of gauge theory and resolving the spectrum of local operators, Phys. Rev. D 78 (2008) 126003 [arXiv:0807.3696] [InSPIRE].

[16] M. Lasalle, An explicit formula for characters of the symmetric group, arXiv:0707.2732.

[17] M. Hayashi, A Group Theoretic Approach to Quantum Information, Springer (2017).

[18] G. Chiribella, G.M. D'Ariano and P. Perinotti, Quantum Circuit Architecture, Phys. Rev. Lett. 101 (2008) 060401.

[19] G. Gutoski and J. Watrous, Toward a general theory of quantum games, in Proceeding of the 39th Annual ACM Symposium on Theory of Computation, San Diego, California, U.S.A., June 11-13, 2007, pp. 565 [DOI:10.1145/1250790.1250873]. 
[20] G. Chiribella, G.M. D'Ariano and P. Perinotti, Theoretical framework for quantum networks, Phys. Rev. A 80 (2009) 022339.

[21] A. Bisio, G. Chiribella, G.M. D'Ariano and P. Perinotti, Quantum Networks: general theory and applications, Acta Phys. Slov. 61 (2011) 273 [arXiv:1601.04864].

[22] D. Bacon, I. Chuang and A. Harrow, Efficient Quantum Circuits for Schur and Clebsch-Gordan Transforms, Phys. Rev. Lett. 97 (2006) 170502 [quant-ph/0407082].

[23] N. Beisert, The complete one loop dilatation operator of $N=4$ superYang-Mills theory, Nucl. Phys. B 676 (2004) 3 [hep-th/0307015] [InSPIRE].

[24] R. de Mello Koch, M. Dessein, D. Giataganas and C. Mathwin, Giant Graviton Oscillators, JHEP 10 (2011) 009 [arXiv:1108.2761] [INSPIRE].

[25] R. de Mello Koch and S. Ramgoolam, A double coset ansatz for integrability in AdS/CFT, JHEP 06 (2012) 083 [arXiv: 1204.2153] [INSPIRE].

[26] V. Balasubramanian, B. Czech, K. Larjo and J. Simon, Integrability versus information loss: A Simple example, JHEP 11 (2006) 001 [hep-th/0602263] [INSPIRE].

[27] J. Simon, Correlations vs connectivity in R-charge, JHEP 10 (2018) 048 [arXiv: 1805.11279] [INSPIRE].

[28] D. Berenstein and A. Miller, Superposition induced topology changes in quantum gravity, JHEP 11 (2017) 121 [arXiv:1702.03011] [INSPIRE].

[29] S. Ryu and T. Takayanagi, Holographic derivation of entanglement entropy from AdS/CFT, Phys. Rev. Lett. 96 (2006) 181602 [hep-th/0603001] [INSPIRE].

[30] M. Van Raamsdonk, Building up spacetime with quantum entanglement, Gen. Rel. Grav. 42 (2010) 2323 [arXiv: 1005. 3035] [INSPIRE].

[31] T. Faulkner, M. Guica, T. Hartman, R.C. Myers and M. Van Raamsdonk, Gravitation from Entanglement in Holographic CFTs, JHEP 03 (2014) 051 [arXiv: 1312.7856] [INSPIRE].

[32] J. Maldacena and L. Susskind, Cool horizons for entangled black holes, Fortsch. Phys. 61 (2013) 781 [arXiv:1306.0533] [INSPIRE].

[33] M. Hamermesh, Group theory and its application to physical problems, Dover (1962). 